arXiv: 1308.5627

\title{
The tension on the cosmological parameters from different observational data
}

\author{
Qing Gad* \\ MOE Key Laboratory of Fundamental Quantities Measurement, School of Physics, \\ Huazhong University of Science and Technology, Wuhan 430074, China \\ Yungui Gongt \\ MOE Key Laboratory of Fundamental Quantities Measurement, School of Physics, \\ Huazhong University of Science and Technology, Wuhan 430074, China and \\ Institute of Theoretical Physics, Chinese Academy of Sciences, Beijing 100190, China
}

\begin{abstract}
Planck measurements of the cosmic microwave background power spectra find a lower value of the Hubble constant $H_{0}$ and a higher value of the fractional matter energy density $\Omega_{m 0}$ for the concordance $\Lambda \mathrm{CDM}$ model, and these results are in tension with other measurements. The Planck group argued that the tension came either from some sources of unknown systematic errors in some astrophysical measurements or the wrong $\Lambda \mathrm{CDM}$ model applied in fitting the data. We studied the reason for the tension on $H_{0}$ from different measurements by considering two dynamical dark energy models. We found that there is no tension between different data, the constraint on $H_{0}$ is almost unchanged for different dark energy models and the tension with the local measurements remains when the error bar on $H_{0}$ is tightened to be around 1 . We argue that the tension on $H_{0}$ is not caused by the fitting model.
\end{abstract}

PACS numbers: 95.36.+x, 98.80.Es

\footnotetext{
* gaoqing01good@163.com

† yggong@mail.hust.edu.cn
} 


\section{INTRODUCTION}

Ever since the discovery of the cosmic acceleration found by the observations of type Ia supernovae (SNe Ia) in 1998 [1-3], an exotic energy component dubbed as dark energy with negative pressure which contributes about $72 \%$ to the total energy density in the universe has been proposed. Alternatively, modified theories of gravity such as the DvaliGabadadze-Porrati model [4], f(R) gravity [5] 7], dRGT ghost-free massive gravity [8, 9], are also used to explain the cosmic acceleration. Although the cosmological constant is the simplest candidate for dark energy and is consistent with current observations, dynamical dark energy models such as the quintessence model [10 14] are also explored due to the many orders of magnitude discrepancy between the theoretical estimation and astronomical observations for the cosmological constant. For a recent review of dark energy, please see Ref. [15-18].

Since the nature of dark energy is still unknown and a self-consistent dark energy theory is still unavailable, current observational data provides us the only possible way to probe the nature of dark energy. A lot of analyses by using the observational data have been done in the literature [19 51]. In particular, one usually parameterizes the equation of state parameter $w(z)$ of dark energy with several parameters, such as the Chevallier-Polarski-Linder (CPL) parametrization $w(a)=w_{0}+w_{a}(1-a)$ with two parameters $w_{0}$ and $w_{a}$ [43, 44] and the SSLCPL model with explicit degeneracy relation between $w_{0}$ and $w_{a}$ [52, 53]. Because of the degeneracies among the parameters $\Omega_{m 0}, w_{0}$ and $w_{a}$ in the model, complementary cosmological observations are needed to break the degeneracies. The measurements on the cosmic microwave background anisotropy, the baryon acoustic oscillation (BAO) measurements and the SNe Ia observations provide complementary data.

Recently, Planck released its first year results which give a higher value of fractional matter energy density $\Omega_{m_{0}}=0.315 \pm 0.017$ and a lower value of the Hubble constant $H_{0}=$ $67.3 \pm 1.2 \mathrm{~km} \mathrm{~s}^{-1} \mathrm{Mpc}^{-1} 1$ for the concordance $\Lambda$ CDM model [54, 55]. However, Tammannk et al gave a lower value $H_{0}=62.3 \pm 1.3$ (random) \pm 4 (systematic) $\mathrm{km} \mathrm{s}^{-1} \mathrm{Mpc}^{-1}$ by using the Cepheid-calibrated luminosity of SNe Ia [56] which differs at about $2.4 \sigma$ from the result $H_{0}=73.8 \pm 2.4 \mathrm{~km} \mathrm{~s}^{-1} \mathrm{Mpc}^{-12}$ by calibrating the magnitude-redshift relation of $253 \mathrm{SNe}$ Ia with over 600 Cepheid variables [57] due to the difference of the calibration of zero points.

\footnotetext{
${ }^{1}$ We refer this value as the Planck result

2 This value is referred as the local measurements
} 
Applying the revised geometric maser distance to NGC 4258 to the same Cepheid data used in [57], the Hubble constant was lowered to be $H_{0}=70.6 \pm 3.3 \mathrm{~km} \mathrm{~s}^{-1} \mathrm{Mpc}^{-1}$ [58]. If strong metallicity prior was imposed and three different distance anchors were combined, the Hubble constant was measured as $H_{0}=72.5 \pm 2.5 \mathrm{~km} \mathrm{~s}^{-1} \mathrm{Mpc}^{-1}$ [58]. By measuring the time delays between multiple images of lensed sources with free-form modelling of gravitational lenses, the Hubble constant was determined to be $H_{0}=69 \pm 6$ (statistic) \pm 4 (systematic) $\mathrm{km} \mathrm{s}^{-1} \mathrm{Mpc}^{-1}$ [59]. It seems that the calibration of zero point of SNe Ia data is still a controversial issue. The Planck result disagrees with the local measurements at about $2.5 \sigma$ level. The Planck group argued that the tension came either from some sources of unknown systematic errors in some astrophysical measurements or the wrong $\Lambda \mathrm{CDM}$ model applied in fitting the data [55]. Despite of the tension on $H_{0}$ for the concordance $\Lambda$ CDM model between the Planck constraint and the local distance ladder measurements with SNe Ia data, the Planck result is consistent with the results from BAO data [60 65] and the Hubble parameter $H(z)$ data [66 69]. This excludes the possibility that the tension is due to the different redshift regions covered by different data. In [70], the authors found that some of the tensions come from the $217 \mathrm{GHz} \times 217 \mathrm{GHz}$ detector set spectrum, and they found $\Omega_{m 0}=0.302 \pm 0.015$ and $H_{0}=68.1 \pm 1.1 \mathrm{~km} \mathrm{~s}^{-1} \mathrm{Mpc}^{-1}$ by using a map-based foreground cleaning procedure. By considering the effect of the cosmic variance on the measurement of the local Hubble constant [71] or additional sterile neutrinos [72], it was shown that the tension can be partially relieved.

For the concordance $\Lambda \mathrm{CDM}$ model, the best fitting value from the three year Supernova Legacy Survey (SNLS3) SNe Ia data [73] is $\Omega_{m 0}=0.227_{-0.035}^{+0.042}$ [55], and the constraint by the Union2.1 SNe Ia data is $\Omega_{m 0}=0.295_{-0.040}^{+0.043}[74]$. With the improved photometric calibration of 740 SNe Ia (JLA data) obtained from the joint analysis of the Sloan Digital Sky Survey (SDSS)-II and SNLS collaborations [75], the constraint becomes $\Omega_{m 0}=0.295 \pm 0.034$ which is consistent with both Union2.1 and the Planck results. It seems that the tension on $\Omega_{m 0}$ between the SNLS3 SNe Ia constraint and the Planck constraint (at about $2 \sigma$ level) is due to the systematics of the calibration of the SNLS3 SNe Ia data, so no such tension from different measurements exists.

However, for different data it is still worthy studying the dependence of the parameter constraints on dynamical dark energy models and their consistencies with $\Lambda \mathrm{CDM}$ model. For the dark energy model with constant equation of state ( $w \mathrm{CDM}$ model), Planck data 
alone gave $\Omega_{m 0}=0.204_{-0.051}^{+0.052}, w=-1.49_{-0.29}^{+0.30}$ and $H_{0}=85.0 \pm 10.9$ [65] which is in tension with the flat $\Lambda$ CDM model with $w=-1$ at more than $1 \sigma$ level. If the $H_{0}$ prior is combined with Planck data, $w=-1.24_{-0.19}^{+0.18}$ at $95 \%$ confidence level which is in tension with the flat $\Lambda \mathrm{CDM}$ model at more than $2 \sigma$ level [55]. Fitting the CPL model to the combined BAO and Planck data, it was found that $w_{0}=-1.04_{-0.69}^{+0.72}$ and $w_{a}<1.32$ at $95 \%$ confidence level, and $\Lambda$ CDM model with $w_{0}=-1$ and $w_{a}=0$ is consistent with both the Planck $+\mathrm{WP}+\mathrm{BAO} 3$ and the Planck+WP+Union2.1 combination but is in tension with the Planck+WP+SNLS combination at about the $2 \sigma$ level [55]. By using a different method of dark energy perturbation, it was found that $\Lambda \mathrm{CDM}$ model is consistent with the data combination of Planck+WP+SNLS+BAO [76]. In [77], the authors considered the CPL model with the addition of dark radiation and the imposing of the $H_{0}=73.8 \pm 2.4$ prior and they found that the concordance $\Lambda \mathrm{CDM}$ model is disfavoured at more than $1 \sigma$ level. Since the discrepancy between the Planck result and the local measurements is at about the $2.5 \sigma$ level, the use of $H_{0}$ prior is inconsistent and the flat prior $([-2,2])$ on $w_{a}$ used in [55, 77] may be too restrictive. We would like to address the issue whether the tension can be relieved by using the dynamical dark energy models.

In this paper, we first apply a one parameter SSLCPL model with explicit relation between $w_{0}$ and $w_{a}$ which models a wide class of thawing scalar field over a large redshift region, then we use the CPL parametrization with the $8 \mathrm{BAO}$ data $[60-64]$ and the 21 Hubble parameter $H(z)$ data [66 69] in combination of three different SNe Ia samples: the SNLS3 sample of 472 SNe Ia data [73], the Union2.1 sample of 580 SNe Ia data [74], and the JLA sample of 780 SNe Ia data from the joint analysis of SNLS and SDSS-II collaborations [75], and the cosmic microwave background anisotropy data from the combination of Planck temperature power spectrum with the WMAP polarization low-multipole likelihood data [54, 55, 78].

\section{OBSERVATIONAL DATA}

The SNLS3 SNe Ia data consist of 123 low-redshift SNe Ia with $z \lesssim 0.1$ mainly from Calan/Tololo, the Harvard-Smithsonian Center for Astrophysics (CfA) survey releases CfAI, CfAII and CfAIII, and Carnegie Supernova Project (CSP), 242 SNe Ia over the redshift range $0.08<z<1.06$ observed from the SNLS [73], 93 intermediate-redshift SNe Ia with

\footnotetext{
3 The meaning of different data combination is explained in the next section
} 
$0.06 \lesssim z \lesssim 0.4$ observed during the first season of SDSS-II supernova survey [79], and 14 high-redshift SNe Ia with $z \gtrsim 0.8$ from the Hubble Space Telescope (HST) [80]. The SNLS3 SNe Ia data used the combination of SALT2 and SiFTO light-curve fitters [73]. We also include the correction on the dependence of the host-galaxy stellar mass. For the fitting to the SNLS3 data, we need to add two more nuisance parameters $\alpha$ and $\beta$ which characterize the stretch-luminosity and color-luminosity relations in addition to the model parameters and the nuisance parameter $\mathcal{M}_{B}$ which accounts for some combination of the absolute magnitude of a fiducial SN Ia and the Hubble constant. Because the nuisance parameter $\mathcal{M}_{B}$ incorporates the absolute magnitude and the Hubble constant, and the normalization of the magnitude is arbitrary, so we marginalize over it in the SNe Ia data fitting process, and $H_{0}$ is not a fitting parameter for the SNe Ia data.

The JLA sample combines the data from SNLS3 and three years of the SDSS-II SNe survey with the joint light-curve analysis by the SDSS-II and SNLS collaborations, and it includes a total of 740 spectroscopically confirmed SNe Ia with the improved photometric calibration [75]. For the fitting to the JLA data, we also need to add two more nuisance parameters $\alpha$ and $\beta$.

The Union2.1 SNe Ia data consists of 580 SNe Ia which augments the Union2 compilation [81] with 14 new SNe Ia from the HST Cluster Supernova Survey [74] and 9 low redshift SNe Ia from the CSP [82], and it uses the SALT2 light-curve fitter. There are 256 SNe Ia contained in both the SNLS3 and Union2.1 compilations.

For the BAO data, we use the measurements from the 6dFGS survey [60], the galaxy clustering in the Baryon Oscillation Spectroscopic Survey (BOSS) [62], the distribution of galaxies in the SDSS survey [61], the WiggleZ dark energy survey [63], and Ly $\alpha$ forest of high-redshift quasars in BOSS survey [64]. Percival et al measured the distance ratio $d_{z}=r_{s}\left(z_{d}\right) / D_{V}(z)$ at two redshifts $z=0.2$ and $z=0.35$ by fitting to the power spectra of luminous red galaxies and main-sample galaxies in the SDSS [61]. Beutler et al derived that $d_{0.106}=0.336 \pm 0.015$ from the 6dFGS measurements [60]. The BOSS survey gave $d_{0.57}^{-1}=13.67 \pm 0.22[62]$. The WiggleZ dark energy survey measured the acoustic parameter $A(z)=D_{V}(z) \sqrt{\Omega_{m} H_{0}^{2}} / z$ at three redshifts $z=0.44, z=0.6$ and $z=0.73$ [63]. Busca et al reported the radial BAO data $\Delta z(z)=H(z) r_{s}\left(z_{d}\right) / c=0.11404 \pm 0.00396$ at the redshift $z=2.3$ by detecting the BAO in the Ly $\alpha$ forest of high-redshift quasars from the BOSS Survey [64]. 
For the Hubble parameter $H(z)$ data, we use the $H(z)$ data at 11 different redshifts obtained from the differential ages of passively evolving galaxies [66, 68], two Hubble parameter data $H(z=0.24)=79.69 \pm 2.65 \mathrm{~km} \mathrm{~s}^{-1} \mathrm{Mpc}^{-1}$ and $H(z=0.43)=86.45 \pm 3.68$ $\mathrm{km} \mathrm{s}^{-1} \mathrm{Mpc}^{-1}$ determined by taking the BAO scale as a standard ruler in the radial direction [67], and the $H(z)$ data at eight different redshifts obtained from the differential spectroscopic evolution of early-type galaxies as a function of redshift [69]. The total number of $H(z)$ data is 21 . Although the quality of the data is not good, but it helps constrain the behaviour of dark energy because it depends on $w(z)$ by its first integral.

For the Planck data [54], we use the Planck temperature power spectrum data together with the nine years of WMAP polarization low-multipole likelihood which was called Planck+WP in [54], hereafter we call this data Planck data for short. For the SSLCPL model discussed in section 3 , a flat prior over the range $[-3,2]$ was assumed for the parameter $w_{0}$. For the CPL model discussed in section 4, we choose the prior ranges $[-3,2]$ for $w_{0}$ and $[-10,3]$ for $w_{a}$. Larger prior ranges were imposed because the likelihood of $w_{0}$ has a long tail with $w_{0}>-0.3$ as can be seen from our results in section 4 .

\section{SSLCPL PARAMETRIZATION}

To study the effect of dynamical dark energy model on the tensions among different data, we first consider the SSLCPL parametrization [52, 53] which approximates the dynamics of general thawing scalar fields [83] over a large redshift range with only one free parameter $w_{0}$, and reduces to $\Lambda \mathrm{CDM}$ model when the parameter $w_{0}=-1$. The model does not differ much from $\Lambda$ CDM model, it approximates a wide class of thawing scalar fields with the equation of state $w(a)=w_{0}+w_{a}(1-a)$, and the parameter $w_{a}$ is a function of $w_{0}$ and $\Omega_{m 0}$. For the flat case, the equation of state of the SSLCPL model is [52, 53]

$$
w(a)=w_{0}+6\left(1+w_{0}\right) \frac{\left(\Omega_{\phi 0}^{-1}-1\right)\left[\sqrt{\Omega_{\phi 0}}-\tanh ^{-1}\left(\sqrt{\Omega_{\phi 0}}\right)\right]}{\Omega_{\phi 0}^{-1 / 2}-\left(\Omega_{\phi 0}^{-1}-1\right) \tanh ^{-1}\left(\sqrt{\Omega_{\phi 0}}\right)}(1-a),
$$

where $\Omega_{\phi 0}=1-\Omega_{m 0}$. By fitting the flat SSLCPL model to the SNLS3 SNe Ia data alone, the Union2.1 SNe Ia data alone, the JLA SNe Ia data alone, the BAO data alone and the $H(z)$ data alone, we find that the values of $\Omega_{m 0}$ are all consistent except that the SNLS3 SNe Ia result is marginally consistent at the $1 \sigma$ level. The SNLS3 SNe Ia data alone prefers smaller values of $\Omega_{m 0}$ and bigger values of $w_{0}$. Next, we combine different SNe Ia data and 
Planck data with the $\mathrm{BAO}$ and $H(z)$ data and the constraints on the model parameters from different combinations are shown in table II and figure 1, By combining the BAO and $H(z)$ data with different SNe Ia and Planck data, we find that the constraints on the parameters are all consistent as seen from table I and figure 1. In particular, the value of $H_{0}$ from different data agrees at the $1 \sigma$ level for the SSLCPL model. The combination of Planck $+\mathrm{BAO}+H(z)$ prefers more negative value of $w_{0}$ which is marginally consistent with $\Lambda$ CDM model at the $1 \sigma$ level, and the error bar on $H_{0}$ is doubled comparing with the constraint on $\Lambda \mathrm{CDM}$ model. The combination of SNLS3+BAO $+H(z)$ gives a lower value of $\Omega_{m 0}$ and a higher value of $H_{0}$ and the result is in tension with $\Lambda$ CDM model. With the improved photometric calibration, the combination of $\mathrm{JLA}+\mathrm{BAO}+H(z)$ not only tightens the constraint on $w_{0}$ a little bit but also eliminates the tension with $\Lambda$ CDM model. Both the combinations of Union $2.1+\mathrm{BAO}+H(z)$ and $\mathrm{JLA}+\mathrm{BAO}+H(z)$ are consistent with $\Lambda \mathrm{CDM}$ model. Furthermore, the constraint on $H_{0}$ from $\mathrm{JLA}+\mathrm{BAO}+H(z)$ is consistent with both the local measurements and the Planck result because of the larger error bar on $H_{0}$.

TABLE I. The marginalized $1 \sigma$ constraints on the flat SSLCPL model.

\begin{tabular}{cccc}
\hline Data & $\Omega_{m 0}$ & $w_{0}$ & $H_{0}$ \\
\hline Union2.1+BAO $+H(z)$ & $0.291_{-0.019}^{+0.018}$ & $-1.03_{-0.12}^{+0.11}$ & $70.5_{-1.9}^{+2.0}$ \\
\hline SNLS3+BAO $+H(z)$ & $0.277_{-0.017}^{+0.018}$ & $-1.12 \pm 0.11$ & $72.1_{-2.0}^{+1.9}$ \\
\hline JLA+BAO+H(z) & $0.292_{-0.019}^{+0.017}$ & $-1.0 \pm 0.09$ & $70.1 \pm 1.7$ \\
\hline Planck+BAO+H(z) & $0.287_{-0.023}^{+0.019}$ & $-1.17_{-0.16}^{+0.17}$ & $70.6_{-2.6}^{+2.7}$ \\
\hline \hline Union2.1+Planck+BAO+H(z) & $0.292_{-0.015}^{+0.013}$ & $-1.12_{-0.11}^{+0.10}$ & $69.9_{-1.8}^{+1.7}$ \\
\hline SNLS3+Planck+BAO+H(z) & $0.287_{-0.013}^{+0.012}$ & $-1.15 \pm 0.09$ & $70.4_{-1.4}^{+1.5}$ \\
\hline JLA+Planck+BAO+H(z) & $0.302_{-0.012}^{+0.011}$ & $-1.04 \pm 0.07$ & $68.6_{-1.2}^{+1.1}$ \\
\hline
\end{tabular}

Since both SNLS3+BAO $+H(z)$ and Planck $+\mathrm{BAO}+H(z)$ prefers lower values of $w_{0}$, when we use the combination of SNLS3+Planck+BAO+H(z), we expect that a tension with $\Lambda \mathrm{CDM}$ model remains, in fact the result is $w_{0}=-1.15 \pm 0.09$ which differs from $\Lambda \mathrm{CDM}$ model at more than $1 \sigma$ level. In addition to this tension, the value of $H_{0}$ is also in tension with the Planck result at about $2 \sigma$ level. Replacing the SNLS3 data with the improved JLA data, we get $\Omega_{m 0}=0.302_{-0.012}^{+0.011}, w_{0}=-1.04 \pm 0.07$ and $H_{0}=68.6_{-1.2}^{+1.1}$ with $\chi^{2}=10518.15$. The constraints on the flat $\Lambda \mathrm{CDM}$ model from the combined JLA + Planck $+\mathrm{BAO}+H(z)$ 

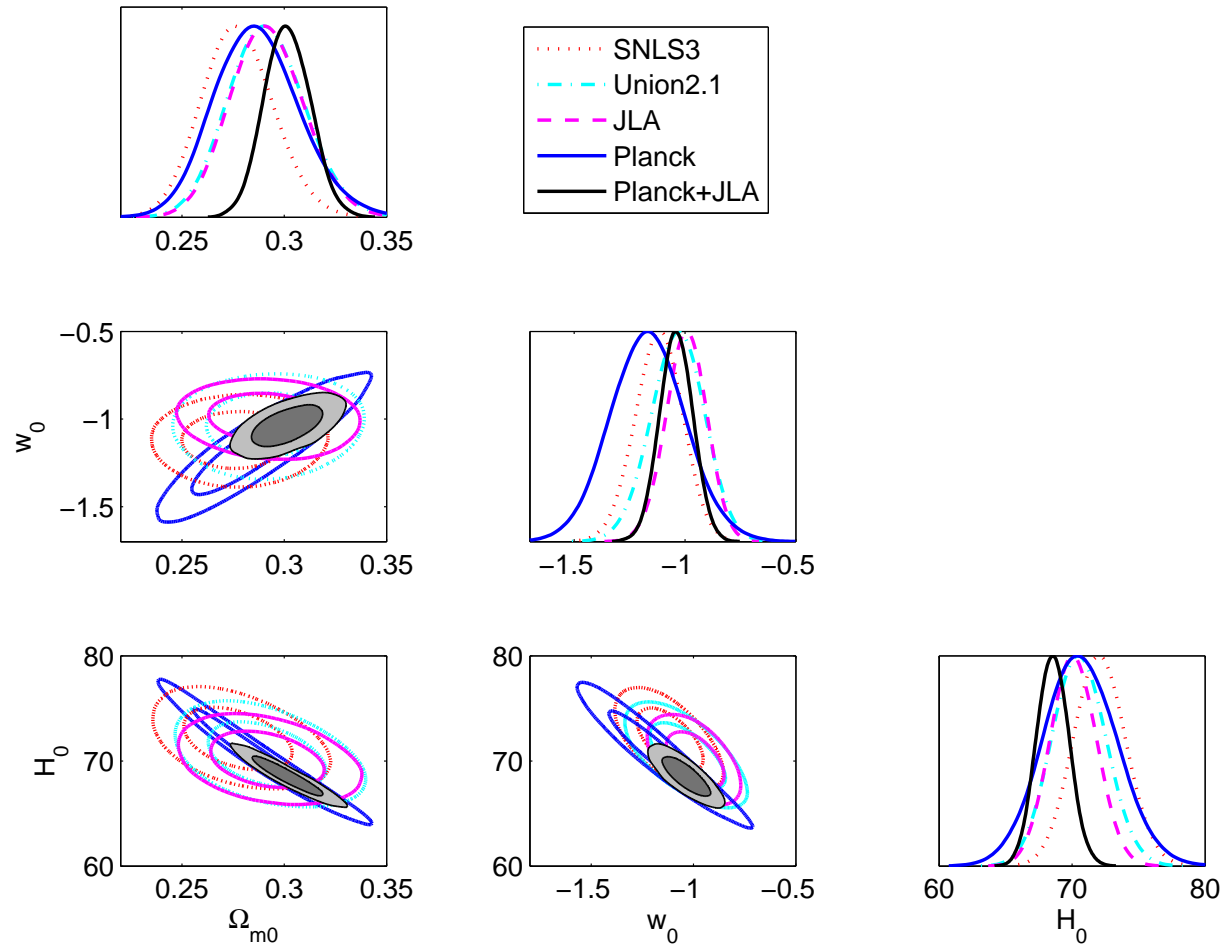

FIG. 1. The marginalized $1 \sigma$ and $2 \sigma$ constraints on the flat SSLCPL model. The red dotted line is for the combination SNLS3+BAO+H(z), the dash-dotted cyan line is for the combination Union2.1 $+\mathrm{BAO}+H(z)$, the dashed magenta line is for the combination $\mathrm{JLA}+\mathrm{BAO}+H(z)$, the solid blue line is for the combination Planck $+\mathrm{BAO}+H(z)$, and the solid black line is the combination $\mathrm{JLA}+\mathrm{Union} 2.1+\mathrm{BAO}+H(z)$.

is $\Omega_{m 0}=0.305 \pm 0.09$ and $H_{0}=68.0_{-0.6}^{+0.7}$ with $\chi^{2}=10518.02$. The results suggest that the systematics in SNLS3 data is the main reason for the tensions. However, the value of $H_{0}=68.6_{-1.2}^{+1.1} \mathrm{~km} \mathrm{~s}^{-1} \mathrm{Mpc}^{-1}$ is now in tension with the local measurements at about $1.9 \sigma$ level although the tension is partially alleviated. Therefore, when dynamical dark energy model is used, the tension on $H_{0}$ may get partially relieved due to larger error bar on $H_{0}$ with the inclusion of more fitting parameters, but $\Lambda$ CDM model seems not to be the reason of the tension. By using the combination of Union2.1+Planck+BAO $+H(z)$, we get $w_{0}=-1.12_{-0.11}^{+0.10}$ which is in tension with $\Lambda$ CDM model at the $1 \sigma$ level. 


\section{CPL PARAMETRIZATION}

Fitting the flat CPL model to the SNLS3 SNe Ia data, we get $\Omega_{m 0}=0.32 \pm 0.08$, $w_{0}=-0.7 \pm 0.3$ and $w_{a}=-4.9_{-4.5}^{+4.2}$ which is marginally consistent with $\Lambda \mathrm{CMD}$ model at the $1 \sigma$ level. Fitting the flat CPL model to the combined Planck+BAO data, we get $\Omega_{m 0}=0.307_{-0.042}^{+0.03}, w_{0}=-0.90_{-0.49}^{+0.37}, w_{a}=-0.76_{-0.94}^{+1.62}$ and $H_{0}=68.9_{-4.0}^{+3.9} \mathrm{~km} \mathrm{~s}^{-1} \mathrm{Mpc}^{-1}$ which is consistent with the flat $\Lambda$ CDM model and those from SNLS3 SNe Ia data alone, the result is also consistent with that in [76]. The combined BAO+Planck data also gives much tighter constraint on the variation of dark energy parameter $w_{a}$ than SNLS3 SNe Ia data due to the tighter constraint on $\Omega_{m 0}$, and it prefers a more negative value of $w_{0}$. The BAO and Planck data do not prefer dark energy models with rapid change of $w(z)$, so they constrain $w_{a}$ to be a smaller range around zero.

Fitting the flat CPL model to the combined SNLS3 $+\mathrm{BAO}+H(z)$ data, we get $\Omega_{m 0}=$ $0.289 \pm 0.020, w_{0}=-0.96 \pm 0.18, w_{a}=-1.13 \pm 1.20$ and $H_{0}=71.9_{-1.9}^{+2.0} \mathrm{~km} \mathrm{~s}^{-1} \mathrm{Mpc}^{-1}$ which differs by about $2 \sigma$ from the Planck result. Fitting the flat CPL model to the combined Union2.1+BAO $+H(z)$ data, we get $\Omega_{m 0}=0.298_{-0.022}^{+0.023}, w_{0}=-0.95_{-0.21}^{+0.17}, w_{a}=-0.58_{-0.75}^{+1.27}$ and $H_{0}=70.3 \pm 2.0$ which are consistent with both the $\Lambda$ CDM model and the Planck result. The constraints from Union2.1 data are also consistent with SNLS3 data. Replacing the SNLS3 data by the improved JLA data, we get $\Omega_{m 0}=0.30 \pm 0.02, w_{0}=-0.89_{-0.17}^{+0.13}$, $w_{a}=-0.82_{-0.75}^{+1.16}$ and $H_{0}=70.0 \pm 1.7$ which is consistent with both SNLS3 and Union2.1 data, and the results are also consistent with $\Lambda$ CDM model. The JLA data prefers larger value of $w_{0}$. Comparing with the constraints on the SSLCPL model, we find that the constraints on $H_{0}$ are almost the same even though we have one more parameter $w_{a}$. Fitting the CPL model

to the combined Placnk $+\mathrm{BAO}+H(z)$ data, we get $\Omega_{m 0}=0.309_{-0.036}^{+0.029}, w_{0}=-0.86_{-0.39}^{+0.34}$, $w_{a}=-0.84_{-0.90}^{+1.23}$ and $H_{0}=68.6_{-3.6}^{+3.5} \mathrm{~km} \mathrm{~s}^{-1} \mathrm{Mpc}^{-1}$ which is consistent with the $\Lambda$ CDM model. The results are summarized in table \1 and shown in figure 2. With one more parameter on $w(z)$, the Placnk+BAO $+H(z)$ data prefers larger value of $\Omega_{m 0}$ and smaller value of $H_{0}$, and the constraints on the cosmological parameters become a little looser. Although the constraints from different SNe Ia data and Planck data are consistent, the JLA data is more compatible with the Planck data than the Union2.1 data, and the JLA data give much tighter constraints than the Planck data as seen from table II and figure 2.

Fitting the flat CPL model to the combined Union2.1+Planck+BAO $+H(z)$ data, we 
TABLE II. The marginalized $1 \sigma$ constraints on CPL model.

\begin{tabular}{ccccc}
\hline Data & $\Omega_{m 0}$ & $w_{0}$ & $w_{a}$ & $H_{0}$ \\
\hline Union2.1+BAO $+H(z)$ & $0.298_{-0.022}^{+0.023}$ & $-0.95_{-0.21}^{+0.17}$ & $-0.58_{-0.75}^{+1.27}$ & $70.3 \pm 2.0$ \\
\hline SNLS3+BAO $+H(z)$ & $0.289 \pm 0.020$ & $-0.96 \pm 0.18$ & $-1.13 \pm 1.20$ & $71.9_{-1.9}^{+2.0}$ \\
\hline JLA+BAO+H(z) & $0.30 \pm 0.02$ & $-0.89_{-0.17}^{+0.13}$ & $-0.82_{-0.75}^{+1.16}$ & $70.0 \pm 1.7$ \\
\hline Planck+BAO+H(z) & $0.309_{-0.036}^{+0.029}$ & $-0.86_{-0.39}^{+0.34}$ & $-0.84_{-0.90}^{+1.23}$ & $68.6_{-3.6}^{+3.5}$ \\
\hline \hline Union2.1+Planck+BAO+H(z) & $0.298_{-0.017}^{+0.015}$ & $-0.98_{-0.2}^{+0.17}$ & $-0.49_{-0.54}^{+0.79}$ & $69.5_{-1.7}^{+1.8}$ \\
\hline SNLS3+Planck+BAO+H(z) & $0.29_{-0.013}^{+0.012}$ & $-1.02_{-0.14}^{+0.13}$ & $-0.51_{-0.48}^{+0.69}$ & $70.5 \pm 1.4$ \\
\hline JLA+Planck+BAO+H $(z)$ & $0.304 \pm 0.011$ & $-0.90_{-0.12}^{+0.11}$ & $-0.68_{-0.44}^{+0.60}$ & $68.8_{-1.2}^{+1.1}$ \\
\hline
\end{tabular}

get $\Omega_{m 0}=0.298_{-0.017}^{+0.015}, w_{0}=-0.98_{-0.2}^{+0.17}, w_{a}=-0.49_{-0.54}^{+0.79}$, and $H_{0}=69.5_{-1.7}^{+1.8} \mathrm{~km} \mathrm{~s}^{-1} \mathrm{Mpc}^{-1}$ which is consistent with $\Lambda \mathrm{CDM}$ model. Fitting the flat CPL model to the combined $\mathrm{SNLS} 3+\mathrm{Planck}+\mathrm{BAO}+H(z)$ data, we get $\Omega_{m 0}=0.290_{-0.013}^{+0.012}, w_{0}=-1.02_{-0.14}^{+0.13}, w_{a}=$ $-0.51_{-0.48}^{+0.69}$, and $H_{0}=70.5 \pm 1.4 \mathrm{~km} \mathrm{~s}^{-1} \mathrm{Mpc}^{-1}$ which differs by about $1.7 \sigma$ from the Planck value for the flat $\Lambda$ CDM model. Fitting the flat CPL model to the combined $\mathrm{JLA}+\mathrm{Planck}+\mathrm{BAO}+H(z)$ data, we get $\Omega_{m 0}=0.304 \pm 0.011, w_{0}=-0.90_{-0.12}^{+0.11}, w_{a}=$ $-0.68_{-0.44}^{+0.60}$, and $H_{0}=68.8_{-1.2}^{+1.1} \mathrm{~km} \mathrm{~s}^{-1} \mathrm{Mpc}^{-1}$ with $\chi^{2}=10516.08$ which is consistent with the results obtained in [75] with distance prior from Planck data. For the Union2.1 data, the results are consistent with $\Lambda$ CDM model due to larger error bars on the constraints. Since the JLA data is more compatible with the Planck data, the combined JLA + Planck $+\mathrm{BAO}+H(z)$ give higher value of $\Omega_{m 0}$ and lower value of $H_{0}$ and the error bars on $\Omega_{m 0}$ and $H_{0}$ are small, the value of $H_{0}$ is consistent with the Planck result, but it is in tension with the local measurements at about $1.9 \sigma$ level. Furthermore, the $\Lambda$ CDM model with $w_{0}=-1$ and $w_{a}=0$ is only marginally consistent with the results from the combined $\mathrm{JLA}+\mathrm{Planck}+\mathrm{BAO}+H(z)$ data at the $1 \sigma$ level.

\section{CONCLUSIONS}

The Planck first year result further supports the concordance $\Lambda$ CDM model with $\Omega_{m 0}=$ $0.315 \pm 0.017$ and $H_{0}=67.3 \pm 1.2 \mathrm{~km} \mathrm{~s}^{-1} \mathrm{Mpc}^{-1}$. Although the tension on $\Omega_{m 0}$ is due to the systematics in SNLS3 SNe Ia data, the tension on $H_{0}$ with the local measurements 

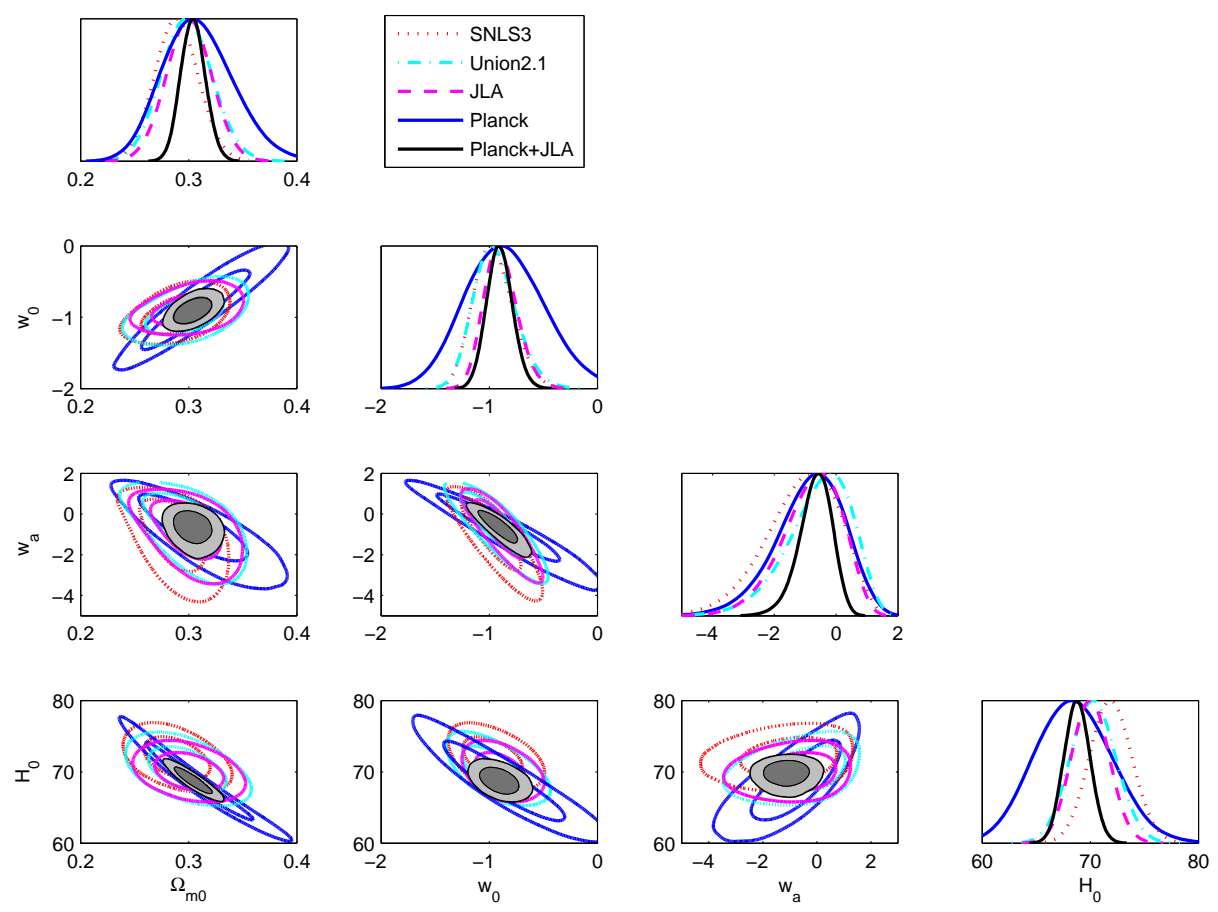

FIG. 2. The marginalized $1 \sigma$ and $2 \sigma$ constraints on the flat CPL model. The red dotted line is for the combination SNLS3+BAO $+H(z)$, the dash-dotted cyan line is for the combination Union2.1+BAO $+H(z)$, the dashed magenta line is for the combination $\mathrm{JLA}+\mathrm{BAO}+H(z)$, the solid blue line is for the combination Planck $+\mathrm{BAO}+H(z)$, and the solid black line is the combination $\mathrm{JLA}+\mathrm{Union} 2.1+\mathrm{BAO}+H(z)$.

still exists. We study the reason behind it by lifting the restriction on the dark energy model. In particular, we extend the flat $\Lambda$ CDM model to flat SSLCPL model and flat CPL model. For the flat SSLCPL model, we find that Planck data is consistent with BAO and $H(z)$ data, and the constraints $\Omega_{m 0}=0.287_{-0.023}^{+0.019}$ and $H_{0}=70.6_{-2.6}^{+2.7} \mathrm{~km} \mathrm{~s}^{-1} \mathrm{Mpc}^{-1}$ from the combined Planck $+\mathrm{BAO}+H(z)$ data are consistent with the local measurements and the Planck results for the concordance $\Lambda$ CDM model, but the value of $w_{0}=-1.17_{-0.16}^{+0.17}$ is marginally consistent with $w_{0}=-1$ at the $1 \sigma$ level. Both the Union2.1 and JLA data are consistent with $\Lambda \mathrm{CDM}$ model and the Planck data, the SNe Ia data give much better constraints on $w_{0}$ than the Planck data. Due to larger error bars on $H_{0}$, the constraints on $H_{0}$ from Union2.1 $+\mathrm{BAO}+H(z), \mathrm{JLA}+\mathrm{BAO}+H(z)$ and $\mathrm{Planck}+\mathrm{BAO}+H(z)$ data are consistent with both the local measurements and the Planck result. Combing the Union2.1 SNe Ia data 
with the Planck data, we get $\Omega_{m 0}=0.292_{-0.015}^{+0.013}, w_{0}=-1.12_{-0.11}^{+0.10}$ and $H_{0}=69.9_{-1.8}^{+1.7}$. The value of $w_{0}$ is in tension with the $\Lambda \mathrm{CDM}$ value $w_{0}=-1$ at the $1 \sigma$ level although the value of $H_{0}$ is consistent with both Planck result and the local measurements due to larger error bars. For the combined JLA + Planck $+\mathrm{BAO}+H(z)$ data, there is no tension with the $\Lambda$ CDM model, but the value of $H_{0}=68.6_{-1.2}^{+1.1}$ is in tension with the local measurements by about $1.9 \sigma$. The result suggests that with reduced error bar on the cosmological parameters due to higher quality of data, the tension on $H_{0}$ remains.

The constraints on SSLCPL model from the combined Planck+BAO+H(z) data are marginally consistent with $\Lambda$ CDM model although the SSLCPL is a simple extension of $\Lambda$ CDM model. Therefore, we further ease the restriction on the dark energy model by considering the flat CPL parametrization. The results show that no tension exists among different data for the flat CPL model and the results from different data in combinations with $\mathrm{BAO}+H(z)$ data are consistent with $\Lambda \mathrm{CDM}$ model. The combined $\mathrm{JLA}+\mathrm{BAO}+H(z)$ data give much tighter constraints on $\Omega_{m 0}, w_{0}$ and $H_{0}$ than the combined Planck $+\mathrm{BAO}+H(z)$ data. Because we have more parameters, the error bars on the parameters are usually larger, that may be the reason for the consistency of the results. The error bars can be further reduced when we combine the SNe Ia data with the Planck data because they have different degeneracy directions. By fitting the flat CPL model to the combined Union2.1+Planck+BAO $+H(z)$ data, we get $\Omega_{m 0}=0.298_{-0.017}^{+0.015}$, $w_{0}=-0.98_{-0.2}^{+0.17}, w_{a}=-0.49_{-0.54}^{+0.79}$, and $H_{0}=69.5_{-1.7}^{+1.8} \mathrm{~km} \mathrm{~s}^{-1} \mathrm{Mpc}^{-1}$ which is consistent with $\Lambda \mathrm{CDM}$ model, but the error bar is still large. Fitting the flat CPL model to the combined JLA + Planck $+\mathrm{BAO}+H(z)$ data, we get $\Omega_{m 0}=0.304 \pm 0.011, w_{0}=-0.90_{-0.12}^{+0.11}$, $w_{a}=-0.68_{-0.44}^{+0.60}$, and $H_{0}=68.8_{-1.2}^{+1.1} \mathrm{~km} \mathrm{~s}^{-1} \mathrm{Mpc}^{-1}$. With the addition of Planck data to the JLA data, we get $w_{a}<0$ at the $1 \sigma$ level, the constraints on $\Omega_{m 0}, w_{a}$ and $H_{0}$ was improved about $40 \%$, the result is only marginally consistent with $\Lambda$ CDM model and $H_{0}$ is in tension with the local measurements. Note that when the distance priors from the Planck data were used, it was found that $w_{a}=-0.336 \pm 0.552$ which is consistent with $\Lambda$ CDM value $w_{a}=0[75]$. Because the constraints on $H_{0}$ are almost unchanged for different SNe Ia data when we use CPL model in place of SSLCPL model, so the tension on $H_{0}$ is not caused by the fitting model. Fitting the combined JLA+Planck+BAO $+H(z)$ data to the flat $\Lambda$ CDM model, we get $\Omega_{m 0}=0.305 \pm 0.09$ and $H_{0}=68.0_{-0.6}^{+0.7}$ which differs about $2.3 \sigma$ from the local measurements. In terms of Akaike information criteria (AIC) [84], $\Lambda$ CDM model is favored. 
From the above analysis, we see that the constraints on $H_{0}$ from SSLCPL and CPL models are almost the same, and the tension on $H_{0}$ with the local measurements exists when the error bar on $H_{0}$ is tightened to be around 1. The tension on $H_{0}$ seems not come from dark energy models, it seems that both the local measurements and the Planck data have some unknown systematics. For example, by using a map-based foreground cleaning procedure based on a combination of $353 \mathrm{GHz}$ and $545 \mathrm{GHz}$ maps, it was found that $H_{0}=68.1 \pm 1.1$ $\mathrm{km} \mathrm{s}^{-1} \mathrm{Mpc}^{-1}$ for the Planck data [70]. By revising the geometric maser distance to NGC 4258, the Hubble constant was lowered to be $H_{0}=70.6 \pm 3.3 \mathrm{~km} \mathrm{~s}^{-1} \mathrm{Mpc}^{-1}$ [58]. So the tension may disappear if systematics were properly accounted for.

In conclusion, there is no tension among different data. With the improved photometric calibration for the SNLS3 SNe Ia data, the JLA data give tighter constraints on the cosmological parameters. The combined $\mathrm{JLA}+\mathrm{BAO}+H(z)$ data constrain $w_{0}$ much better than the combined Planck $+\mathrm{BAO}+H(z)$ data, and the combined $\mathrm{JLA}+\mathrm{Planck}+\mathrm{BAO}+H(z)$ data constrains $H_{0}$ to be better than $2 \%$. Although dynamical dark energy model with $w_{a}<0$ is preferred at the $1 \sigma$ level by the combined JLA+Planck+BAO $+H(z)$ data, $\Lambda$ CDM model is still favored in term of AIC. The tension on $H_{0}$ with the local measurements remains and it is not caused by the fitting model. Further studies on the systematics of both Planck data and the local measurements on $H_{0}$ are needed.

\section{ACKNOWLEDGMENTS}

This work was partially supported by the National Basic Science Program (Project 973) of China under grant No. 2010CB833004, the NNSF of China under grant nos. 10935013 and 11175270, the Program for New Century Excellent Talents in University under grant no. NCET-12-0205 and the Fundamental Research Funds for the Central Universities under grant no. 2013YQ055.

[1] Perlmutter S et al. (Supernova Cosmology Project) 1998 Nature 391 51-54 (arXiv: astro$\mathrm{ph} / 9712212)$

[2] Riess A G et al. (Supernova Search Team) 1998 Astron. J. 116 1009-1038 (arXiv: astro$\mathrm{ph} / 9805201)$ 
[3] Perlmutter S et al. (Supernova Cosmology Project) 1999 Astrophys. J. 517 565-586 (arXiv: astro-ph/9812133)

[4] Dvali G, Gabadadze G and Porrati M 2000 Phys. Lett. B. 485 208-214 (arXiv: hepth/0005016)

[5] Carroll S M, Duvvuri V, Trodden M and Turner M S 2004 Phys. Rev. D 70043528 (arXiv: astro-ph/0306438)

[6] Starobinsky A A 2007 JETP Lett. 86 157-163 (arXiv: 0706.2041)

[7] Hu W and Sawicki I 2007 Phys. Rev. D 76064004 (arXiv: 0705.1158)

[8] de Rham C, Gabadadze G and Tolley A J 2011 Phys. Rev. Lett. 106231101 (arXiv: 1011.1232)

[9] Gong Y 2013 Commun. Theor. Phys. 59 319-323 (arXiv: 1207.2726)

[10] Ratra B and Peebles P 1988 Phys. Rev. D 373406

[11] Wetterich C 1988 Nucl. Phys. B 302668

[12] Caldwell R, Dave R and Steinhardt P J 1998 Phys. Rev. Lett. 80 1582-1585 (arXiv: astro$\mathrm{ph} / 9708069)$

[13] Zlatev I, Wang L M and Steinhardt P J 1999 Phys. Rev. Lett. 82 896-899 (arXiv: astro$\mathrm{ph} / 9807002)$

[14] Steinhardt P J, Wang L M and Zlatev I 1999 Phys. Rev. D 59123504 (arXiv: astro$\mathrm{ph} / 9812313)$

[15] Sahni V and Starobinsky A A 2000 Int. J. Mod. Phys. D 09 373-444 (arXiv: astro$\mathrm{ph} / 9904398)$

[16] Copeland E J, Sami M and Tsujikawa S 2006 Int. J. Mod. Phys. D. 15 1753-1936 (arXiv: hep-th/0603057)

[17] Padmanabhan T 2008 Gen. Rel. Grav. 40 529-564 (arXiv: 0705.2533)

[18] Li M, Li X D, Wang S and Wang Y 2011 Commun. Theor. Phys. 56 525-604 (arXiv: $1103.5870)$

[19] Alam U, Sahni V, Saini T D and Starobinsky A 2004 Mon. Not. Roy. Astron. Soc. 354275 (arXiv: astro-ph/0311364)

[20] Alam U, Sahni V and Starobinsky A 2004 JCAP 0406008 (arXiv: astro-ph/0403687)

[21] Barger V, Gao Y and Marfatia D 2007 Phys. Lett. B 648 127-132 (arXiv: astro-ph/0611775)

[22] Clarkson C, Cortes M and Bassett B A 2007 JCAP 0708011 (arXiv: astro-ph/0702670)

[23] Corasaniti P S and Copeland E 2003 Phys. Rev. D 67063521 (arXiv: astro-ph/0205544) 
[24] Astier P 2001 Phys. Lett. B 500 8-15 (arXiv: astro-ph/0008306)

[25] Efstathiou G 1999 Mon. Not. Roy. Astron. Soc. 310 842-850 (arXiv: astro-ph/9904356)

[26] Gerke B F and Efstathiou G 2002 Mon. Not. Roy. Astron. Soc. 33533 (arXiv: astro$\mathrm{ph} / 0201336)$

[27] Sullivan S, Cooray A and Holz D E 2007 JCAP 0709004 (arXiv: 0706.3730)

[28] Wang Y and Mukherjee P 2004 Astrophys. J. 606 654-663 (arXiv: astro-ph/0312192)

[29] Weller J and Albrecht A 2001 Phys. Rev. Lett. 86 1939-1942 (arXiv: astro-ph/0008314)

[30] Huang Q G, Li M, Li X D and Wang S 2009 Phys. Rev. D. 80083515 (arXiv: 0905.0797)

[31] Shafieloo A, Sahni V and Starobinsky A A 2009 Phys. Rev. D. 80101301 (arXiv: 0903.5141)

[32] Lampeitl H, Nichol R, Seo H, Giannantonio T, Shapiro C et al. 2009 Mon. Not. Roy. Astron. Soc. 401 2331-2342 (arXiv: 0910.2193)

[33] Serra P, Cooray A, Holz D E, Melchiorri A, Pandolfi S et al. 2009 Phys. Rev. D. 80121302 (arXiv: 0908.3186)

[34] Gong Y, Cai R G, Chen Y and Zhu Z H 2010 JCAP 1001019 (arXiv: 0909.0596)

[35] Gong Y, Wang B and Cai R g 2010 JCAP 1004019 (arXiv: 1001.0807)

[36] Pan N, Gong Y, Chen Y and Zhu Z H 2010 Class. Quant. Grav. 27155015 (arXiv: 1005.4249)

[37] Gong Y, Zhu X m and Zhu Z H 2011 Mon. Not. Roy. Astron. Soc. 4151943 (arXiv: 1008.5010)

[38] Gong Y, Gao Q and Zhu Z H 2013 Mon. Not. Roy. Astron. Soc. 430 3142-3154 (arXiv: $1110.6535)$

[39] Li X D, Li S, Wang S, Zhang W S, Huang Q G et al. 2011 JCAP 1107011 (arXiv: 1106.4116)

[40] Li Z, Wu P and Yu H 2011 Phys. Lett. B 695 1-8 (arXiv: 1011.1982)

[41] Su Q, Tuo Z L and Cai R G 2011 Phys. Rev. D 84103519 (arXiv: 1109.2846)

[42] Wetterich C 2004 Phys. Lett. B. 594 17-22 (arXiv: astro-ph/0403289)

[43] Chevallier M and Polarski D 2001 Int. J. Mod. Phys. D. 10 213-224 (arXiv: gr-qc/0009008)

[44] Linder E V 2003 Phys. Rev. Lett. 90091301 (arXiv: astro-ph/0208512)

[45] Jassal H, Bagla J and Padmanabhan T 2005 Mon. Not. Roy. Astron. Soc. 356 L11-L16 (arXiv: astro-ph/0404378)

[46] Barboza E, Alcaniz J, Zhu Z H and Silva R 2009 Phys. Rev. D 80043521 (arXiv: 0905.4052)

[47] Farooq O, Mania D and Ratra B 2013 Astrophys. J. 764138 (arXiv: 1211.4253)

[48] Farooq O and Ratra B 2013 Astrophys. J. 766 L7 (arXiv: 1301.5243)

[49] Zhang S N and Ma Y Z 2013 arXiv: 1303.6124 
[50] Yahya S, Seikel M, Clarkson C, Maartens R and Smith M 2014 Phys. Rev. D 89023503 (arXiv: 1308.4099)

[51] Benitez-Herrera S, Ishida E, Maturi M, Hillebrandt W, Bartelmann M et al. 2013 arXiv: 1308.5653

[52] Gao Q and Gong Y 2013 Int. J. Mod. Phys. D 221350035 (arXiv: 1212.6815)

[53] Gong Y and Gao Q 2014 Eur. Phys. J. C 742729 (arXiv: 1301.1224)

[54] Ade P et al. (Planck Collaboration) 2013 arXiv: 1303.5062

[55] Ade P et al. (Planck Collaboration) 2013 arXiv: 1303.5076

[56] Tammann G, Sandage A and Reindl B 2008 Astron. Astrophys. Rev. 15 289-331 (arXiv: 0806.3018)

[57] Riess A G, Macri L, Casertano S, Lampeitl H, Ferguson H C et al. 2011 Astrophys. J. 730 119 (arXiv: 1103.2976)

[58] Efstathiou G 2013 arXiv: 1311.3461

[59] Sereno M and Paraficz D 2013 arXiv: 1310.2251

[60] Beutler F, Blake C, Colless M, Jones D H, Staveley-Smith L et al. 2011 Mon. Not. Roy. Astron. Soc. 416 3017-3032 (arXiv: 1106.3366)

[61] Percival W J et al. (SDSS Collaboration) 2010 Mon. Not. Roy. Astron. Soc. $4012148-2168$ (arXiv: 0907.1660)

[62] Anderson L, Aubourg E, Bailey S, Bizyaev D, Blanton M et al. 2013 Mon. Not. Roy. Astron. Soc. 427 3435-3467 (arXiv: 1203.6594)

[63] Blake C, Kazin E, Beutler F, Davis T, Parkinson D et al. 2011 Mon. Not. Roy. Astron. Soc. 418 1707-1724 (arXiv: 1108.2635)

[64] Busca N G et al. (BOSS COllaboration) 2013 Astron. Astrophys. 552 A96 (arXiv: 1211.2616)

[65] Addison G E, Hinshaw G and Halpern M 2013 Mon. Not. Roy. Astron. Soc. 436 1674-1683 (arXiv: 1304.6984)

[66] Simon J, Verde L and Jimenez R 2005 Phys. Rev. D. 71123001 (arXiv: astro-ph/0412269)

[67] Gaztanaga E, Cabre A and Hui L 2009 Mon. Not. Roy. Astron. Soc. 399 1663-1680 (arXiv: 0807.3551)

[68] Stern D, Jimenez R, Verde L, Kamionkowski M and Stanford S A 2010 JCAP 1002008 (arXiv: 0907.3149)

[69] Moresco M, Cimatti A, Jimenez R, Pozzetti L, Zamorani G et al. 2012 JCAP 1208006 (arXiv: 
1201.3609)

[70] Spergel D, Flauger R and Hlozek R 2013 arXiv: 1312.3313

[71] Marra V, Amendola L, Sawicki I and Valkenburg W 2013 Phys. Rev. Lett. 110241305 (arXiv: 1303.3121)

[72] Wyman M, Rudd D H, Vanderveld R A and Hu W 2014 Phys. Rev. Lett. 112051302 (arXiv: 1307.7715)

[73] Conley A, Guy J, Sullivan M, Regnault N, Astier P et al. 2011 Astrophys. J. Suppl. 1921 (arXiv: 1104.1443)

[74] Suzuki N, Rubin D, Lidman C, Aldering G, Amanullah R et al. 2012 Astrophys. J. 74685 (arXiv: 1105.3470)

[75] Betoule M, Kessler R, Guy J, Mosher J, Hardin D et al. 2014 arXiv: 1401.4064

[76] Xia J Q, Li H and Zhang X 2013 Phys. Rev. D 88063501 (arXiv: 1308.0188)

[77] Cheng C and Huang Q G 2014 Phys. Rev. D 89043003 (arXiv: 1306.4091)

[78] Bennett C et al. (WMAP) 2013 Astrophys. J. Suppl. 20820 (arXiv: 1212.5225)

[79] Kessler R, Becker A, Cinabro D, Vanderplas J, Frieman J A et al. 2009 Astrophys. J. Suppl. 185 32-84 (arXiv: 0908.4274)

[80] Riess A G, Strolger L G, Casertano S, Ferguson H C, Mobasher B et al. 2007 Astrophys. J. 659 98-121 (arXiv: astro-ph/0611572)

[81] Amanullah R, Lidman C, Rubin D, Aldering G, Astier P et al. 2010 Astrophys. J. $716712-738$ (arXiv: 1004.1711)

[82] Contreras C, Hamuy M, Phillips M, Folatelli G, Suntzeff N B et al. 2010 Astron. J. 139 519-539 (arXiv: 0910.3330)

[83] Caldwell R and Linder E V 2005 Phys. Rev. Lett. 95141301 (arXiv: astro-ph/0505494)

[84] Akaike H 1974 IEEE Transactions on Automatic Control 19 716-723 